\title{
Computational metabolism modeling predicts risk of distant relapse-free survival in breast cancer patients
}

\section{Computational metabolism modeling predicts risk of distant relapse-free survival in breast cancer patients}

Lucía Trilla-Fuertes ${ }^{1 \uparrow}$, Angelo Gámez-Pozo ${ }^{1,2 \pi}$, Mariana Díaz- Almirón ${ }^{3,}$ Guillermo PradoVázquez ${ }^{1}$, Andrea Zapater-Moros ${ }^{2}$, Rocío López-Vacas ${ }^{2}$, Paolo Nanni ${ }^{4}$, Pilar Zamora ${ }^{5}$, Enrique Espinosa ${ }^{5}$, and Juan Ángel Fresno Vara ${ }^{2 *}$.

${ }^{1}$ Biomedica Molecular Medicine SL, C/ Faraday 7, 28049, Madrid, Spain

${ }^{2}$ Molecular Oncology \& Pathology Lab, Institute of Medical and Molecular Genetics-INGEMM, La Paz University Hospital-IdiPAZ, Paseo de la Castellana 261, 28046, Madrid, Spain

${ }^{3}$ Biostatistics Unit, La Paz University Hospital-IdiPAZ, Paseo de la Castellana 261, 28046, Madrid. Spain

${ }^{4}$ Functional Genomics Centre Zurich, University of Zurich/ETH Zurich, Winterthurerstrasse 190, 8057 , Zurich, Switzerland.

${ }^{5}$ Medical Oncology Service, La Paz University Hospital-IdiPAZ, Paseo de la Castellana 261, 28046, Madrid, Spain

"These authors contribute equally to this work

* Corresponding author

e-mails: LT-F: lucia.trilla@biomedicamm.com; AG-P: angelo.gamez@idipaz.es; MD-A: manadiaz2@gmail.com; GP-V: 14gprado@gmail.com; AZ-M: andreazapater@hotmail.com; RLV: rlvacas@gmail.com; PN: paolo.nanni@fgcz.uzh.ch; PZ: zamorapilar@gmail.com; EE: eespinosa00@hotmail.com; JAFV: juanangel.fresno@salud.madrid.org 


\section{Abstract}

Aims: Differences in metabolism among breast cancer subtypes suggest that metabolism plays an important role in this disease. Flux Balance Analysis is used to explore these differences as well as drug response.

Materials \& Methods: Proteomics data from breast tumors were obtained by massspectrometry. Flux Balance Analysis was performed to study metabolic networks. Flux activities from metabolic pathways were calculated and used to build prognostic models.

Results: Flux activities of vitamin A, tetrahydrobiopterin and beta-alanine metabolism pathways split our population into low- and high-risk patients. Additionally, flux activities of glycolysis and glutamate metabolism split triple negative tumors into low- and high-risk groups.

Conclusions: Flux activities summarize Flux Balance Analysis data and can be associated with prognosis in cancer.

Keywords: breast cancer, metabolism, prognosis, flux balance analysis, personalized medicine 


\section{Introduction}

Breast cancer has a high incidence, with 266,120 estimated new cases and 40,920 estimated deaths in women in the United States during 2018 [1]. The expression of estrogen receptor, progesterone receptor and human epidermal growth factor receptor 2 (HER2) classifies breast cancer into one of three groups: hormone receptor positive / HER2 negative (ER+), HER2 positive (HER2+) or triple negative (TNBC). In previous works, we defined a new subtype within $E R+$ tumors with a clinical outcome and molecular features more similar to TNBC. This new subtype was called TN-like. ER+ tumors which still have ER+ characteristics were renamed as ER-true [2].

Reprogramming of metabolism is a hallmark of cancer [3]. Tumor cells use glucose to produce lactate, thus avoiding glucose metabolism through the Krebs cycle [4]. Tumor cells also produce lactate from glutamine and then generate NADPH in a process known as glutaminolysis [5]. However, not all tumors show the same metabolic alterations. In previous studies we described that there are differences in metabolism between breast cancer subtypes [6].

Proteomics provides detailed information about biological processes. Technical improvements currently allow the quantification of thousands of proteins. This information can be used to calculate the output of metabolic pathways with Flux Balance Analysis (FBA). FBA is a computational method used to study metabolic networks, making possible to predict tumor growth rate or the rate of production of a metabolite [7]. In our previous study, preliminary results of FBA were shown. FBA predicted a higher growth rate in TN-like tumors than in ERtrue. Moreover, tumor growth predictions for TNBC and TN-like were comparable. We have proposed the calculation of flux activities as a summary measurement of flux distributions and showed that flux activities can be used to compare metabolic patterns between tumors or cells [8].

The aim of this work is to study in depth the metabolic differences previously characterized in breast cancer $[2,6]$. More specifically, proteomics data were analyzed through FBA to find metabolic pathways with prognostic value. 


\section{Materials and Methods}

Patient cohort

One hundred and six formalin-fixed paraffin-embedded (FFPE) samples from I+12 Biobank and from IdiPAZ Biobank, both integrated in the Spanish Hospital Biobank Network (RetBioH; www.redbiobancos.es), were analyzed. This study was approved by the Ethical Committees of Hospital 12 de Octubre and Hospital La Paz.

Samples were selected according to these criteria: 1) node-positive disease, 2) no HER2 overexpression, and 3) patients treated with adjuvant chemotherapy and hormonal therapy in the case of ER+ tumors. The following clinical data were recorded: patient's age, tumor size, lymph node status, tumor grade, adjuvant therapy administered and distant metastasis-free survival.

\section{Protein isolation}

Proteins were isolated from FFPE samples as described in previous works [6,9]. Briefly, FFPE slices were deparaffinized in xylene and washed with absolute ethanol. Extracts were prepared in $2 \%$ SDS buffer using a protocol based on heat-induced antigen retrieval [10]. Protein concentration was determined using the MicroBCA Protein Assay Kit (Pierce-Thermo Scientific). Then, protein extracts were digested by trypsin and SDS was removed. Samples were dried and resolubilized in $15 \mu \mathrm{L}$ of a $0.1 \%$ formic acid and $3 \%$ acetonitrile solution.

Mass-spectrometry experiments and protein identification

Samples were analyzed on a LTQ-Orbitrap Velos hybrid mass spectrometer (Thermo Fischer Scientific, Bremen, Germany) coupled to NanoLC-Ultra system (Eksigent Technologies, Dublin, CA, USA). Peptides were separated on a self-made column. Solvent composition was $0.1 \%$ formic acid for channel $\mathrm{A}$, and $0.1 \%$ formic acid and $99.9 \%$ acetonitrile for channel $\mathrm{B}$. The massspectrometer was in data-dependent mode, acquiring full-scan spectra at a resolution of 30 , 000 at $400 \mathrm{~m} / \mathrm{z}$ after accumulation to a target value of 1,000,000, followed by CID (collisioninduced dissociation) fragmentation on the twenty most intense signals per cycle.

The acquired data was analyzed by MaxQuant (version 1.2.7.4), and protein identification was done using Andromeda. Oxidation (M), deamidation (N, Q) and N-terminal protein acetylation was set as modifications. Protein abundance was calculated based on the normalized spectra intensity (LFQ). 
Proteomics data were transformed into log2 and missing values were imputed to a normal distribution using Perseus [11]. Additionally, quality criteria as presence in at least $75 \%$ of the samples of each type or at least two unique peptides were required.

Proteomics data is publicly available in Chorus repository (http://chorusproject.org) under the name "Breast Cancer Proteomics".

Flux Balance Analysis and flux activities

FBA was performed using COBRA Toolbox available for MATLAB and the whole human metabolic reconstruction Recon2 $[12,13]$. The biomass reaction proposed in the Recon2 was used as an objective function to quantify tumor growth rate. Gene-Protein-Reaction rules (GPR), which relate genes and proteins with the enzymes involved in the reactions contained into the Recon2, were solved using a modification of the algorithm by Barker et al. [14]. Briefly, "OR" expressions are treated as a sum and "AND" expressions are calculated as the minimum $[2,8]$. Then, GPR values were introduced into the model using a modified E-flux consisted on normalize GPR data using a normal distribution formula [15].

Flux activities were calculated as in previous works [8]. Briefly, the flux activities were the sum of fluxes for all the reactions included in a metabolic pathway defined in the Recon2.

\section{Statistical analyses}

Distant metastasis-free survival was selected as the prognostic variable. Statistical analyses were performed using GraphPad Prism v6 and predictors for distant metastasis-free survival was built using BRB Array Tool, developed by Dr. Richard Simon's team. Cox regression models were done in SPSS IBM Statistics v20. 


\section{Results}

Patient characteristics

One hundred and six samples were selected for the study. Finally, ninety-six patients diagnosed of breast cancer were analyzed. This cohort had been used in previous works from our group $[2,6]$.

After performing the analyses of the 96 samples, of the $71 \mathrm{ER}+$ samples, 50 were reclassified as ER-true and 21 as TN-like, and twenty-six were TNBC tumors [2].

\section{Proteomics experiments}

One hundred and six patients were recruited in this study. Four samples did not have enough protein amounts to perform mass-spectrometry (MS) experiments. After MS experiments, ninety-six samples had useful protein expression data. 3,239 proteins were measured. Quality criteria and filters were applied and a total of 1,095 proteins had two unique peptides and were detected in at least $75 \%$ of the samples of one type, i.e. ER+ or TNBC.

\section{Flux activities}

FBA is a computational method used to study metabolic networks. The whole metabolic human reconstruction Recon 2 was used to perform these analyses. The Recon 2 is composed by 7,440 reactions and 5,063 metabolites grouped in 101 different metabolic pathways.

Using the results from FBA, flux activities were calculated as the sum of fluxes for the reactions included in each metabolic pathway described in the Recon2. Comparing breast cancer subtypes, significant differences in nucleotide interconversion pathway and reactive-oxygen species (ROS) detoxification were found between ER-true and TNBC and between ER-true and TN-like respectively (Figure 1).

Prognostic signatures using flux activities

In order to identify flux activities related with distant relapse-free survival, BRB Array Tool from Dr. Richard Simon was used. We found five flux activities related with distant relapse-free survival (Sup Table 1).

Using these flux activities, a predictor of distant metastasis-free survival was constructed. This signature included the flux activity of three different pathways: vitamin A metabolism, tetrahydrobiopterin metabolism and beta-alanine metabolism. The predictor split our 
population into high-risk and low-risk groups ( $p$-value $=0.0032, H R=6.520$, cut-off $=30 \%-70 \%$ )

(Figure 2, Table 2).

Strikingly, the prognostic signature retained its prognostic value in the ER+ tumors, being the differences statistically significant in the ER-true group ( $p$-value=0.0179), but have worse performance in TNBC tumors (Figure 3).

Multivariate Cox regression model showed that this signature added information to clinical data (Sup Table 2).

Given that TNBC tumors have differences in metabolism when compared with ER+; a predictive signature only for TNBC was built. This signature was formed by the flux activities of glycolysis and glutamate metabolism and split TNBC population into a low- and a high-risk group ( $p$-value $=0.1064, \mathrm{HR}=4.600$, cut-off $=30-70 \%$ ); however, it did not reach significance (Figure 4, Table 3).

In this case, multivariate analysis was not significant (Sup Table 3). 


\section{Discussion}

Alterations in metabolism constitute a hallmark of cancer [3]. We previously described differences in metabolism between breast cancer subtypes [2,6]. TNBC and TN-like tumors had a higher growth rate than ER-true tumors in FBA predictions [2]. In the present work, our aim was to characterize additional differences in metabolic pathways between breast cancer subtypes.

The analysis of metabolism can use data coming both from genomics or proteomics.

Proteomics provides more direct information about biological effectors than genomics, what is more useful in the GPR estimation. Proteomics experiments detected 3,239 proteins in the present study, although strict filtering criteria reduced the number to 1,095 proteins for analysis.

Comparing flux patterns in large cohorts is challenging. Flux activities are a method previously proposed to compare metabolic pathways between control cells and cells treated with drugs targeting metabolism [8]. Now, using flux activities we characterized differences in ROS detoxification and nucleotide interconversion pathways among breast tumor subtypes. Oxidative stress has been related with tumor aggressiveness in ER+ tumors [16]. TNBC tumors also have a high presence of ROS [17]. As expected, our model predicted lower ROS detoxification in ER-true tumors. Nucleotide interconversion category comprehends all the information about ATP and GTP metabolism. Differences in ATP or GTP production between breast cancer subtypes have not been previously described in the literature.

It was also possible to associate these flux activities with distant metastasis-free survival in this cohort. We found some flux activities related with distant relapse-free survival and built a predictor. The predictive signature included the flux activity of three metabolic pathways: vitamin $\mathrm{A}$, tetrahydrobiopterin and beta-alanine metabolism. To our knowledge, this is the first time that data from FBA are associated with prognosis in cancer. Vitamin A or retinol has been related with risk of relapse in breast cancer [18], but there were no previous information about the prognostic value of tetrahydrobiopterin or beta-alanine metabolism. Additionally, the prognostic value of the predictor remained among ER+ tumors

It is well-known that TNBC tumors have differences in metabolism comparing with ER+ tumors $[2,6]$. For this reason, a predictor taking into account only TNBC tumors was done. This signature included flux activities of glycolysis and glutamate metabolism. However, survival and multivariate analyses are not significant, probably due to the small number of samples. An 
increase in glucose uptake and lactate production associated to glycolysis was previously described in TNBC cells [6] It is also well-known that lactate dehydrogenase $B$ and other glycolytic genes are upregulated in TNBC tumors as compared with the other subtypes and that LDHB overexpression is associated with poor clinical outcome [19]. On the other hand, TNBC tumors have a deregulation of glutaminolysis and exhibited the most frequent expression of proteins related with glutamine metabolism than other subtypes [20,21].

The study has some limitations. A validation of the predictors in an independent cohort is needed. Another limitation is the number of samples of each group in this cohort. It is also necessary to validate these findings into larger cohorts of patients. On the other hand, further improvements in mass-spectrometry techniques now allow the detection of more proteins, which would provide further information at GPR level.

\section{Conclusions}

In conclusion, flux activities, method proposed in previous works, now demonstrated its utility in summarizing FBA data and allows its association with prognosis. Differences in ROS detoxification and nucleotide interconversion pathways between breast cancer subtypes were characterized. Moreover, vitamin A, beta-alanine and tetrahydrobiopterin metabolism flux activities could be used to predict risk of distant metastasis-free survival in breast cancer patients. Finally, glycolysis and glutamate metabolism may be used to predict distant relapsefree survival in TNBC tumors.

\section{Summary points}

- Flux activities could be used to characterize differences in metabolic pathways between groups of tumors and to associate them with clinical outcomes.

- There are differences in flux activities of nucleotide interconversion and ROS detoxification between breast cancer subtypes.

- Flux activities of vitamin A, beta-alanine metabolism and tetrahydrobiopterin metabolism predict distant metastasis-free survival in breast cancer patients.

- Flux activities of glycolysis and glutamate metabolism may predict distant relapse-free survival in TNBC. 


\section{References}

1. Siegel RL, Miller KD, Jemal A. Cancer statistics, 2018. CA Cancer J Clin, 68(1), 7-30 (2018).

2. Gámez-Pozo A, Trilla-Fuertes L, Berges-Soria J et al. Functional proteomics outlines the complexity of breast cancer molecular subtypes. Scientific Reports, 7(1), 10100 (2017).

3. Hanahan D, Weinberg RA. Hallmarks of cancer: the next generation. Cell, 144(5), 646674 (2011).

4. Warburg O. The metabolism of carcinoma cells. J Cancer Res, 9, 148-163 (1925).

5. DeBerardinis RJ, Mancuso A, Daikhin E et al. Beyond aerobic glycolysis: transformed cells can engage in glutamine metabolism that exceeds the requirement for protein and nucleotide synthesis. Proc Natl Acad Sci U S A, 104(49), 19345-19350 (2007).

6. Gámez-Pozo A, Berges-Soria J, Arevalillo JM et al. Combined label-free quantitative proteomics and microRNA expression analysis of breast cancer unravel molecular differences with clinical implications. (Ed.^(Eds) (Cancer Res, 2015) 2243-2253.

7. Orth J, Thiele I, Palsson B. What is flux balance analysis? (Ed.^ ${ }^{\wedge}$ (Eds) (Nat Biotechnol, 2010) 245-248.

8. Trilla-Fuertes L, Gámez-Pozo A, Arevalillo JM et al. Molecular characterization of breast cancer cell response to metabolic drugs. Oncotarget, 9(11), 9645-9660 (2018).

9. Gámez-Pozo A, Ferrer NI, Ciruelos E et al. Shotgun proteomics of archival triplenegative breast cancer samples. Proteomics Clin Appl, 7(3-4), 283-291 (2013).

10. Gámez-Pozo A, Sánchez-Navarro I, Calvo E et al. Protein phosphorylation analysis in archival clinical cancer samples by shotgun and targeted proteomics approaches. Mol Biosyst, 7(8), 2368-2374 (2011).

11. Tyanova S, Temu T, Sinitcyn P et al. The Perseus computational platform for comprehensive analysis of (prote)omics data. Nat Methods, 13(9), 731-740 (2016).

12. Schellenberger J, Que R, Fleming R et al. Quantitative prediction of cellular metabolism with constraint-based models: the COBRA Toolbox v2.0. (Ed.^(Eds) (Nature Protocols, 2011) $1290-1307$.

13. Thiele I, Swainston N, Fleming RM et al. A community-driven global reconstruction of human metabolism. Nat Biotechnol, 31(5), 419-425 (2013).

14. Barker BE, Sadagopan N, Wang Y et al. A robust and efficient method for estimating enzyme complex abundance and metabolic flux from expression data. Comput Biol Chem, 59 Pt B, 98-112 (2015).

15. Colijn C, Brandes A, Zucker J et al. Interpreting expression data with metabolic flux models: Predicting Mycobacterium tuberculosis mycolic acid production. (Ed.^(Eds) (PLOS Comput Bio, 2009)

16. Mahalingaiah PK, Ponnusamy L, Singh KP. Chronic oxidative stress causes estrogenindependent aggressive phenotype, and epigenetic inactivation of estrogen receptor alpha in MCF-7 breast cancer cells. Breast Cancer Res Treat, 153(1), 41-56 (2015).

17. Pelicano $\mathrm{H}$, Zhang $\mathrm{W}$, Liu J et al. Mitochondrial dysfunction in some triple-negative breast cancer cell lines: role of mTOR pathway and therapeutic potential. Breast Cancer Res, 16(5), 434 (2014).

18. Formelli F, Meneghini E, Cavadini E et al. Plasma retinol and prognosis of postmenopausal breast cancer patients. Cancer Epidemiol Biomarkers Prev, 18(1), 4248 (2009).

19. McCleland ML, Adler AS, Shang Y et al. An integrated genomic screen identifies LDHB as an essential gene for triple-negative breast cancer. Cancer Res, 72(22), 5812-5823 (2012). 
20. Kim S, Kim DH, Jung WH, Koo JS. Expression of glutamine metabolism-related proteins according to molecular subtype of breast cancer. Endocr Relat Cancer, 20(3), 339-348 (2013).

21. Lampa M, Arlt H, He Tet al. Glutaminase is essential for the growth of triple-negative breast cancer cells with a deregulated glutamine metabolism pathway and its suppression synergizes with mTOR inhibition. PLoS One, 12(9), e0185092 (2017).

Financial disclosure: This work was supported by Instituto de Salud Carlos III, Spanish Economy and Competitiveness Ministry, Spain and co-funded by the FEDER program, "Una forma de hacer Europa" [PI15/01310]; LT-F is supported by the Spanish Economy and Competitiveness Ministry [DI-15-07614]; GP-V is supported by the Consejería de Educación, Juventud y Deporte of Comunidad de Madrid [IND2017/BMD7783]; AZ-M is supported by Jesús Antolín Garciarena fellowship from IdiPAZ. The funders had no role in the study design, data collection and analysis, decision to publish or preparation of the manuscript.

Conflicts of interest: JAFV, EE and AG-P are shareholders in Biomedica Molecular Medicine SL. LT-F and GP-V are employees of Biomedica Molecular Medicine SL. The other authors declare that they have no competing interests.

Ethical conduct: The study was approved by the Ethical Committee of Hospital 12 de Octubre and Hospital La Paz.

\section{Figure and table legends}

Table 1: Patient characteristics.

Table 2: Weights assigned to each metabolic pathway contained in the predictor signature for all tumors.

Table 3: Weights assigned to each metabolic pathway contained in the predictor signature for TNBC tumors.

Figure 1: Flux activities with significant differences between breast cancer subtypes ${ }^{*}=0.01$ to 0.05 , significant; ${ }^{* *}=0.0001$ to 0.001 , extremely significant).

Figure 2: Predictive signature based on the flux activities of vitamin A metabolism, tetrahydrobiopterin metabolism and beta-alanine metabolism. 
bioRxiv preprint doi: https://doi.org/10.1101/468595; this version posted November 12,2018 . The copyright holder for this preprint (which was not certified by peer review) is the author/funder. All rights reserved. No reuse allowed without permission.

Figure 3: Predictor based on beta-alanine, tetrahydrobiopterin and vitamin A flux activities in the different breast cancer subtypes.

Figure 4: Predictor of distant relapse-free survival in TNBC tumors based on the flux activities of glycolysis and glutamate metabolism. 
Nucleotide interconversion

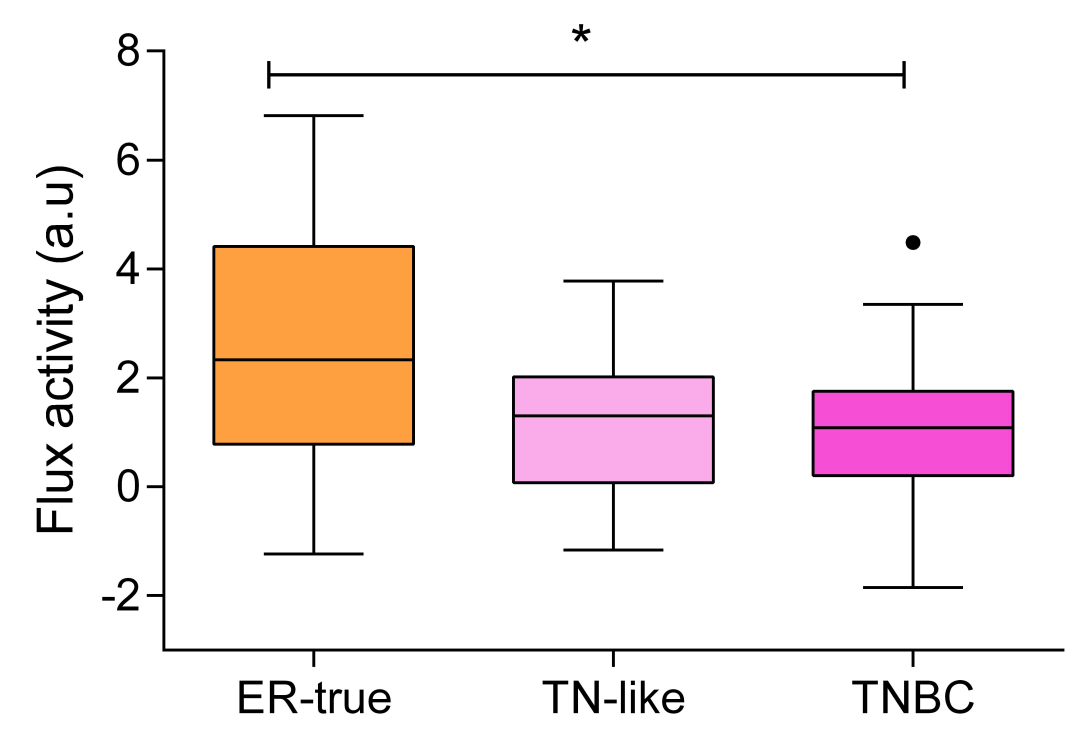

ROS detoxification

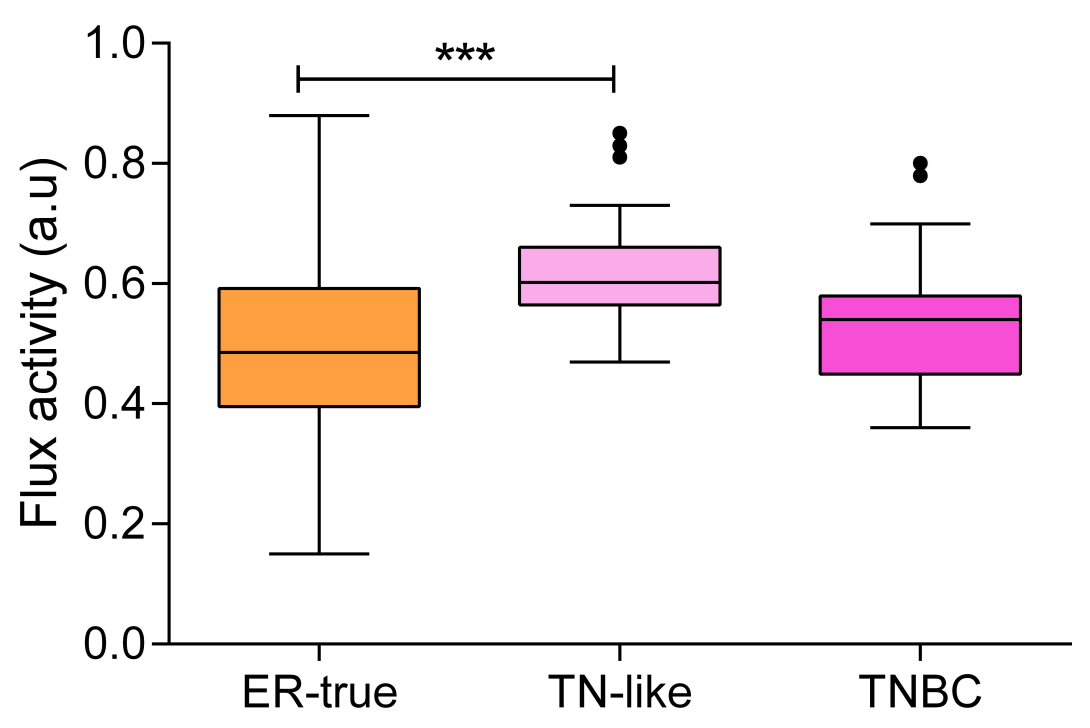




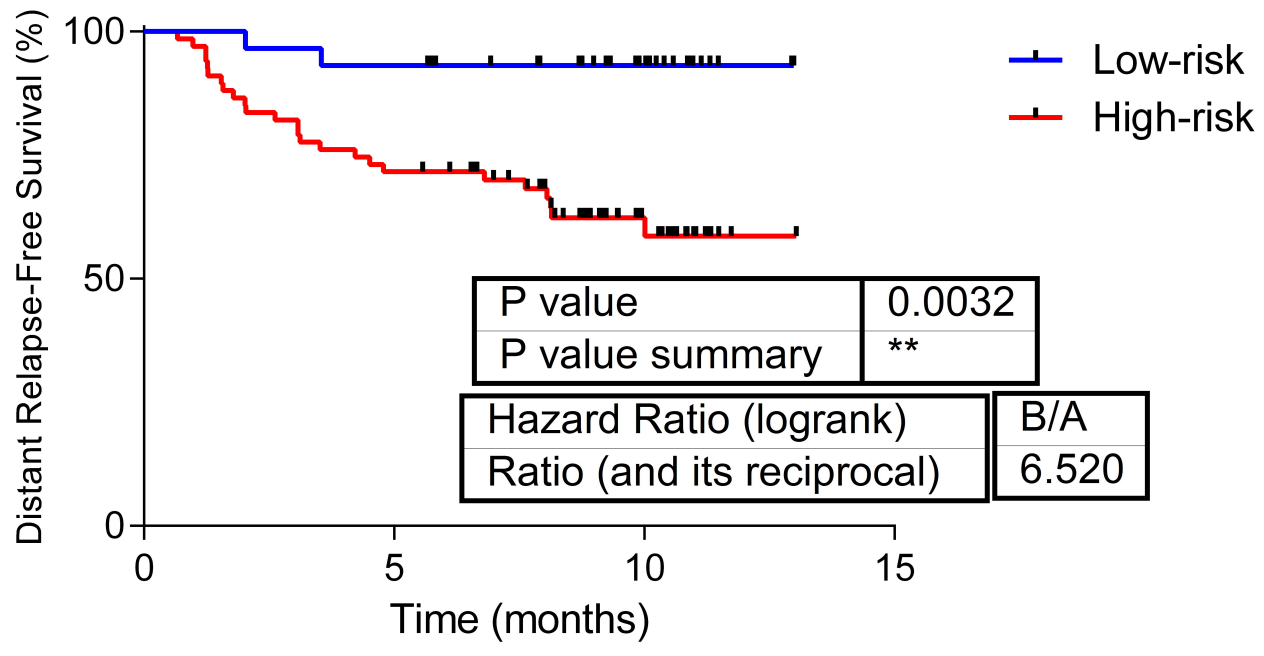


ER-true

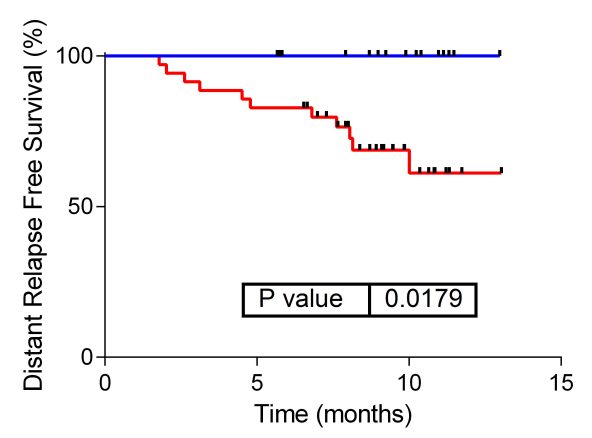

TN-like

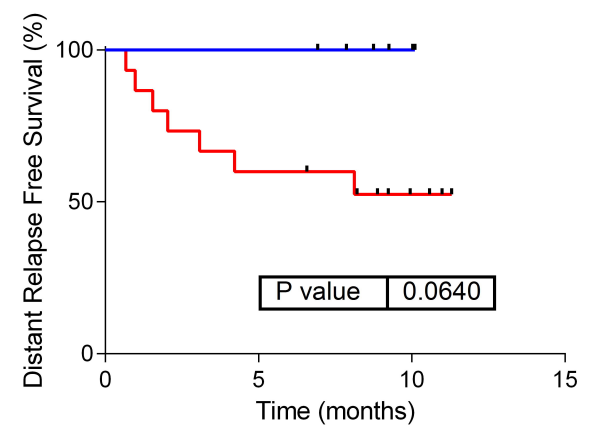

TNBC

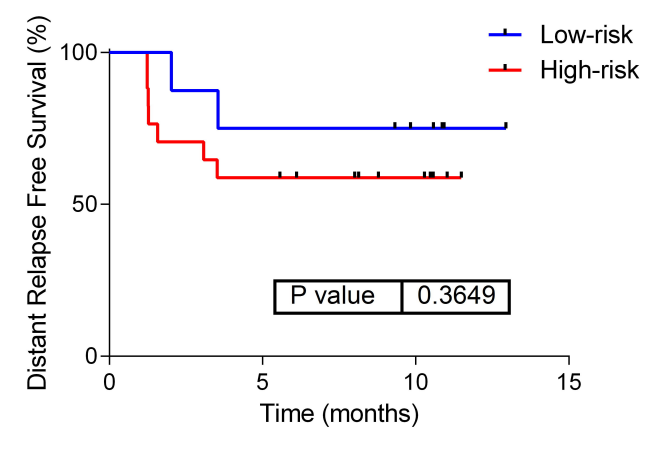




\section{TNBC}

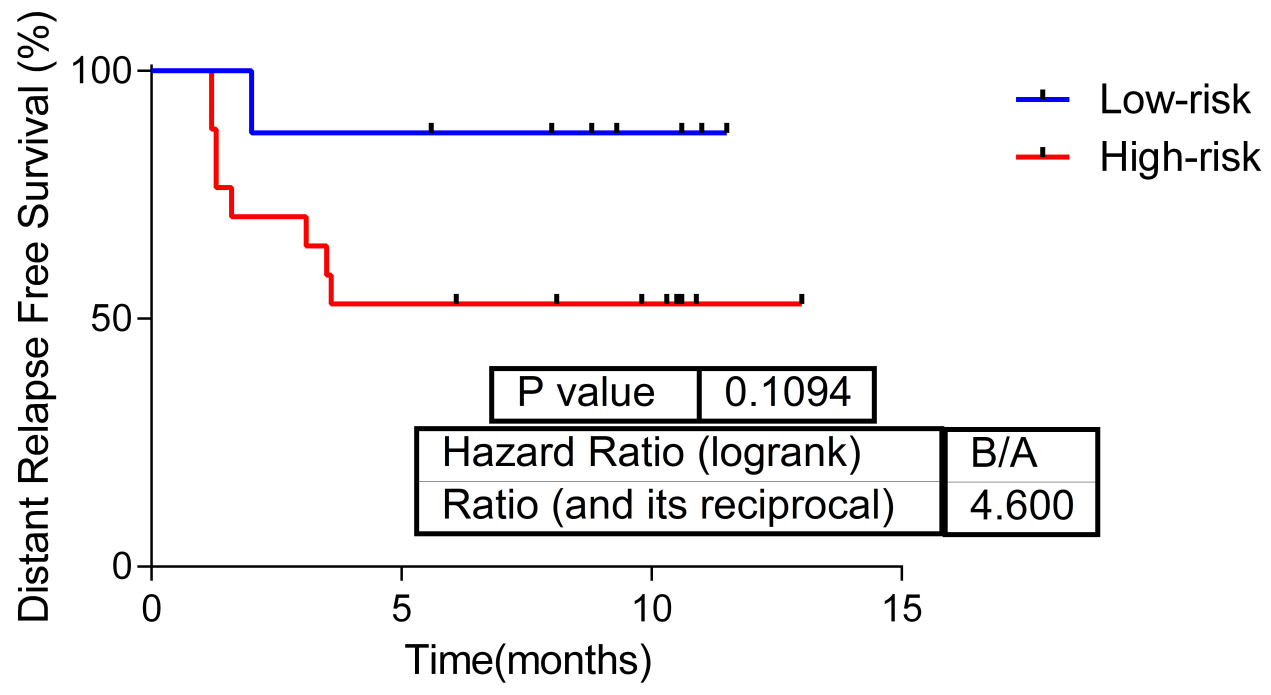

